\title{
MicroRNA-663b mediates TAM resistance in breast cancer by modulating TP73 expression
}

\author{
HUA JIANG ${ }^{*}$, LIN CHENG $^{*}$, PAN HU and RENBIN LIU \\ Breast Cancer Center, Department of Breast and Thyroid Surgery, The Third Affiliated Hospital of Sun Yat-sen University, \\ Guangzhou, Guangdong 510630, P.R. China
}

Received December 14, 2017; Accepted May 9, 2018

DOI: $10.3892 / \mathrm{mmr} .2018 .9064$

\begin{abstract}
Breast cancer is the second leading cause of cancer-associated mortalities in women. Tamoxifen (TAM) is an endocrine therapy commonly used in the treatment of patients with breast cancer expressing estrogen receptor $\alpha$. However, treatment often ends in failure due to the emergence of drug resistance. MicroRNAs (miRNAs), a family of small non-coding RNAs, serve critical roles in the regulation of gene expression and cell events. To date, whether miRNA-663b could mediate TAM resistance in breast cancer remains unknown. Therefore, the aim of the present study was to investigate the role of miRNA-663b in TAM resistance in breast cancer. The results demonstrated that miRNA-663b was upregulated in breast cancer with TAM resistance. Tumor protein 73 (TP73) was a direct target of miRNA-663b, and was negatively regulated by miRNA-663b in MCF-7 cells. Furthermore, it was identified that downregulation of miRNA-663b inhibited cell proliferation ability and promoted cell apoptosis, resulting in enhanced TAM sensitivity. In addition, these findings suggested that TP73 silencing may have eliminated the effects of miRNA-663b inhibitor on breast cancer cells. In conclusion, the present study verified a novel molecular link between miRNA-663b and TP73, and indicated that miRNA-663b may be a critical therapeutic target in breast cancer.
\end{abstract}

\section{Introduction}

Breast cancer is the most common cancer in women worldwide, with about 1.2 million newly diagnosed cases each year (1). Based on the expression of hormone receptors, breast cancer

Correspondence to: Dr Renbin Liu, Breast Cancer Center, Department of Breast and Thyroid Surgery, The Third Affiliated Hospital of Sun Yat-sen University, 600 Tianhe Road, Guangzhou, Guangdong 510630, P.R. China

E-mail: liurb1214@163.com

${ }^{*}$ Contributed equally

Key words: microRNA-663b, breast cancer, tumor protein 73, tamoxifen resistance can be divided into three sub-types: Progesterone receptor (PR), estrogen receptor (ER), and human epidermal growth receptor 2 (HER2). About two-thirds of breast cancer patients show high levels of ER. Tamoxifen (TAM), a selective estrogen receptor $\alpha(\mathrm{ER} \alpha)$ modulator, is the most commonly used endocrine therapy (2-4). TAM adjuvant therapy has been shown to reduce the recurrence and mortality of ER $\alpha$ positive breast cancer patients (5). However, 30-40\% of the patients eventually fail in TAM therapy, and almost all patients with metastatic disease eventually develop into TAM resistance (6-8). Unfortunately, the emergence of resistance to TAM in the treatment of tumors remains a poorly understood and serious clinical problem.

MicroRNA (MiRNA) is a class of endogenous non-coding single-stranded small RNAs of about 18-24 nucleotides, and it regulates the expression of the endogenous genes at transcriptional or post-transcriptional levels by specific binding to the 3'-UTR of target mRNA (9-11). At present, abnormal expression of miRNA are observed in almost all of the malignant tumors, and it is considered that miRNAs are closely related to the occurrence and development of tumors $(12,13)$. A variety of miRNAs have been identified to be abnormally expressed in breast cancer, and play critical roles in the development of breast cancer $(14,15)$. Furthermore, increasing evidence have showed that various miRNAs play an important role in mediating TAM resistance in breast cancer. MiRNA-214 has been reported to increase the sensitivity of breast cancer cells to TAM via inhibiting autophagy by targeting UCP2 (16). MiRNA-155 mediates TAM resistance through regulating SOCS6-STAT3 signal pathway in breast cancer (17). Abnormally low expression of miRNA-342 in breast cancer closely correlates with TAM resistance (18). MiRNA-378a-3p-dependent GOLT1A expression contributes to TAM resistance in breast cancer (19). MiRNA-519a confers TAM resistance by targeting a network of tumour-suppressor genes in breast cancer (20).

To date, whether miRNA-663b could mediate TAM resistance in breast cancer remains unclear. Therefore, the present study aimed to investigate the role and the underlying molecular mechanism of miRNA-663b in TAM resistance in breast cancer.

\section{Materials and methods}

Clinical samples. The present study protocol was approved by the Ethics Committee of The Third Affiliated Hospital of Sun 
Yat-sen University, and written informed consent was obtained from each patient. Human breast cancer tissues with TAM resistance (15 cases) and the control tissues (15 cases) were obtained during the surgery at The Third Affiliated Hospital of Sun Yat-sen University between March 2015 and March 2016. Patients were diagnosed according to the pathological evidence. All the specimens were immediately snap-frozen in liquid nitrogen and then stored at $-80^{\circ} \mathrm{C}$. None of the patients received treatment (chemotherapy or radiotherapy) before surgery.

Cell lines and culture. Human breast cancer cell line MCF7 (expressing ER $\alpha$ ), and the TAM resistant breast cancer cell line MCF7/TAM (expressing ER $\alpha$ ) were purchased from American Type Culture Collection (ATCC, Manassas, VA, USA). MCF7 cells were cultured in RPMI-1640 medium (Gibco; Thermo Fisher Scientific, Inc., Waltham, MA, USA) supplemented with $10 \%$ fetal bovine serum (FBS), $1 \%$ penicillin-streptomycin solution. MCF7/TAM cells were cultured in RPMI-1640 medium supplemented with $10 \%$ fetal bovine serum (FBS), $1 \%$ penicillin-streptomycin solution, and $5 \mathrm{mM}$ TAM (Sigma-Aldrich; Merck KGaA, Darmstadt, Germany). All cells were incubated in a humidified incubator at $37^{\circ} \mathrm{C}$ with $5 \% \mathrm{CO}_{2}$.

Cell transfection. The day before cell transfection, MCF-7 or MCF7/TAM cells $\left(5 \times 10^{4}\right.$ cells/well) were seeded into a 6-well plate and grown in standard conditions. Then MCF-7 or MCF7/TAM cells were transfected with miRNA-663b mimic, mimic control, miRNA-663b inhibitor, inhibitor control, control-shRNA+miRNA-663b inhibitor or TP73-shRNA+miRNA-663b inhibitor with 30 $\mu$ lLipofectamine 2000 reagent (Invitrogen; Thermo Fisher Scientific, Inc.) in line with the manufacturer's protocol. Cells without any treatment were considered as the control group. After incubation for $48 \mathrm{~h}$, these cells were subjected to following experiments. Transfection efficiency was calculated by RT-qPCR.

MTT assay. $48 \mathrm{~h}$ after transfection, MTT assay was carried out to determine the cell proliferation ability. Briefly, MCF7/TAM cells were collected and then reseeded into a 96-well culture plate and cultured in RPMI-1640 medium containing $5 \mathrm{mM}$ TAM at $37^{\circ} \mathrm{C}$ for $24 \mathrm{~h}$, then MTT solution (Amresco, LLC, Solon, OH, USA) was added into every culture well and incubated for another $4 \mathrm{~h}$. Finally, the optical density (OD) value was measured at $450 \mathrm{~nm}$ using a SynergyTM 2 Multi-function microplate reader (BioTek Instruments, Inc., Winooski, VT, USA). Experiments were repeated at least for three times.

Apoptosis analysis assays. MCF7/TAM cells were transfected with miRNA-663b inhibitor, inhibitor control, control-shRNA+miRNA-663b inhibitor or TP73-shRNA+miRNA-663b inhibitor respectively, $48 \mathrm{~h}$ after the transfection, the cells were treated with $5 \mathrm{mM}$ TAM for $48 \mathrm{~h}$ and then labeled with annexin V-FITC and propidium iodide (PI) (Cell Signaling Technology, Inc., Danvers, MA, USA) per as the manufacturer's instructions. Flow cytometry (BD Biosciences, Franklin Lakes, NJ, USA) was applied to analyze cell apoptosis. Tests were repeated three times.

Western blot analysis. After specific treatment, the total cell proteins from MCF7/TAM cells were extracted using lysis
Table I. Primer sequences for polymerase chain reaction.

\begin{tabular}{ll}
\hline Primer & \multicolumn{1}{c}{ Sequence (5'-3') } \\
\hline $\begin{array}{l}\text { miR-663b-Forward } \\
\text { miR-663b-Reverse }\end{array}$ & CGCTAACAGTCTCCAGTC \\
TP73-Forward & CCACCACTTTGAGGTCACTTT \\
TP73-Reverse & CTTCAAGAGCGGGGAGTACG \\
P53-Forward & CCCCTCCTGGCCCCTGTCATCTTC \\
P53-Reverse & GCAGCGCCTCACAACCTCCGTCAT \\
P21-Forward & GACACCACTGGAGGGTGACT \\
P21-Reverse & CAGGTCCACATGGTCTTCCT \\
Bcl2-Forward & TCGCCCTGTGGATGACTGA \\
Bc12-Reverse & CAGAGACAGCCAGGAGAAATC \\
Bax-Forward & TGGCAGCTGACATGTTTTCTGAC \\
Bax-Reverse & TCACCCAACCACCCTGGTCTT \\
GAPDH-Forward & CTTTGGTATCGTGGAAGGACTC \\
GAPDH-Reverse & GTAGAGGCAGGGATGATGTTCT \\
U6-Forward & CTCGCTTCGGCAGCACA \\
U6-Reverse & AACGCTTCACGAATTTGCGT
\end{tabular}

miR, microRNA; TP73, tumor protein 73; Bcl2, B-cell lymphoma 2; Bax, Bcl-2-associated $\mathrm{X}$ protein.

buffer (Cell Signaling Technology, Inc.) according to the manufacturer's protocol. Equal amount of the samples $(25 \mu \mathrm{g})$ were resolved by $10 \%$ SDS-PAGE and then transferred onto polyvinylidene difluoride membranes. After blocking with 5\% non-fat milk at room temperature for $2 \mathrm{~h}$, the membranes were blotted overnight with the primary antibody against TP73 (cat no. 14620), p53 (cat no. 2527), p21 (cat no. 2947), Bcl-2 (cat no. 4223) and Bax (cat no. 5023) (dilution: 1:1,000; Cell Signaling Technology, Inc.) at $4^{\circ} \mathrm{C}$, and then incubated with a secondary antibody. $\beta$-actin (cat no. 4970; dilution: 1:5,000; Cell Signaling Technology, Inc.) was used as the internal control. Finally, the protein bands were observed using the enhanced chemiluminescence detection system (Super Signal West Dura Extended Duration Substrate; Pierce; Thermo Fisher Scientific, Inc.).

Reverse transcription-quantitative polymerase chain reaction (RT-qPCR). Total RNA from cells was extracted using TRIzol regent (Invitrogen; Thermo Fisher Scientific, Inc.) per as the manufacturer's protocol. cDNAs were synthesized by performing reverse transcription experiment using PrimeScript reverse transcription reagent kit (Takara Biotechnology Co., Ltd., Dalian, China) following the manufacturer's instructions. Subsequently, qPCR was applied to analyze the cDNAs and the TaqMan Universal PCR Master Mix kit (Thermo Fisher Scientific, Inc.) was used. Amplification conditions were: $95^{\circ} \mathrm{C}$ for $10 \mathrm{~min}$, followed by 38 cycles of $95^{\circ} \mathrm{C}$ for $10 \mathrm{sec}$ and $58^{\circ} \mathrm{C}$ for $60 \mathrm{sec}$. U6 and GAPDH were used as the internal control for miRNA and mRNA expression respectively. The $2^{-\Delta \Delta \mathrm{Cq}}$ method was applied to quantify the relative gene expressions (21). All the primer sequences for qPCR were constructed by Shanghai Genechem Co., Ltd., (Shanghai, China) and listed in Table I. 
A

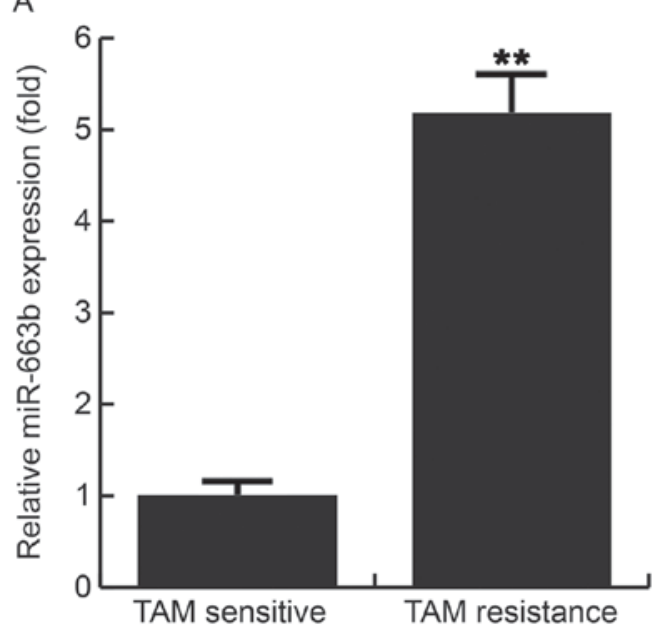

B

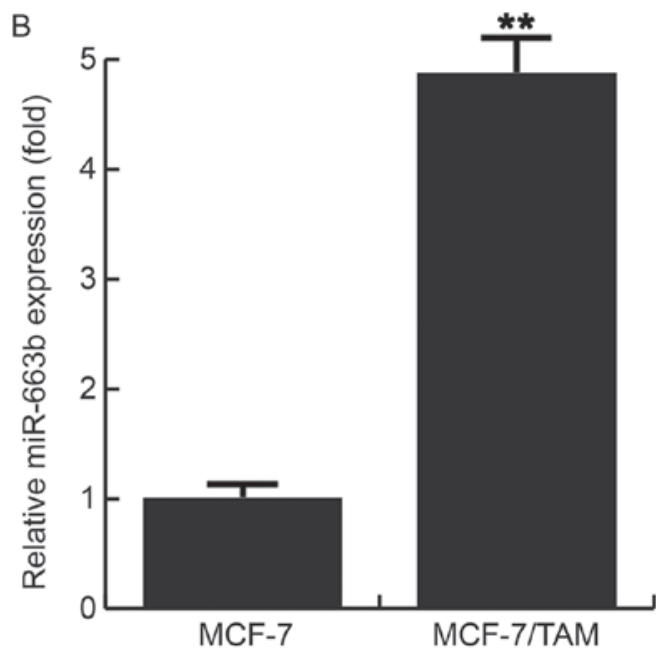

Figure 1. MiRNA-663b expression is upregulated in TAM resistant breast cancer. Reverse transcription-quantitative polymerase chain reaction was used to measure the miRNA-663b expression in the (A) TAM sensitive breast cancer patients and TAM non-sensitive breast cancer patients, and in (B) the human breast cancer cell line MCF7 and the TAM resistant breast cancer cell line MCF7/TAM. Data are presented as the mean \pm standard deviation of 3 repeated experiments. ${ }^{* *} \mathrm{P}<0.01$ vs. TAM sensitive or MCF-7. miRNA/miR, microRNA; TAM, tamoxifen.

Dual luciferase reporter assay. Bioinformatics software (www.targetscan.org) was carried out to predict the potential targets of miRNA-663b, and the findings showed that TP73 was a potential target of miRNA-663b. To confirm our prediction, MCF-7 cells were seeded into each well of a 24 -well plate $\left(5 \times 10^{4}\right.$ cells), 24 h later, the cells were then co-transfected with wild type (WT) or mutant (MUT) TP73 3'UTRpmirGLO plasmid and miRNA-663b mimic or mimic control vector using Lipofectamine 2000 reagent according to the manufacturer's instructions. After incubation for another $48 \mathrm{~h}$, the relative luciferase activity was assessed using the dual-luciferase reporter assay system (Promega Corporation, Madison, WI, USA).

Statistical analysis. Data were presented as the mean \pm standard deviation. SPSS v17.0 statistical software (SPSS, Inc., Chicago, IL, USA) was performed for all statistical analyses. Student's t-test or one-way analysis of varaince followed by Tukey's test was used to make comparison between groups. $\mathrm{P}<0.05$ was considered to indicate a statistically significant difference.

\section{Results}

MiRNA-663b expression is up-regulated in TAM resistant breast cancer. MiRNA-663b expression was detected in breast cancer tissues from TAM non-sensitive and sensitive patients. ER positive patients were treated with TAM and patients with good therapeutic effects were classified as TAM sensitive, and others as TAM resistance. We found that compared with the TAM sensitive patients, miRNA-663b was significantly up-regulated in TAM resistance patients (Fig. 1A). Furthermore, the level of miRNA-663b in human breast cancer cell line MCF7 (expressing ER $\alpha$ ), and the TAM resistant breast cancer cell line MCF7/TAM (expressing $\mathrm{ER} \alpha$ ) was determined, and the findings suggested that MCF7 cells expressed lower levels of miRNA-663b whereas the MCF7/TAM cells exhibited dramatically increased levels of miRNA-663b (Fig. 1B).
MiRNA-663b directly targets TP73. Bioinformatics software (http://www.targetscan.org) was carried out to predict the potential targets of miRNA-663b, and the findings showed that miRNA-663b has hundreds of potential target genes including TP73 which has three miRNA-663b binding sites. Evidence have indicated that TP73 (p73) plays a key role of tumor suppressor though binding and trans-activating of p53-responsive genes and inducing apoatosis and cell arrest. Therefore, we choose TP73 for further analysis. Then, dual luciferase reporter assay was used to confirm the bindings of miRNA-663b with TP73. The findings showed that compared with cells co-transfected with TP73-WT (position 72-76 TP73 3'UTR) and mimic control (miR-C), the relative LUC activity of cells co-transfected with TP73-WT (position 72-76 TP73 3'UTR) and miRNA-663bb was significantly reduced. Besides, (position 1058-1064 TP73 3'UTR) and (position 1560-1566 TP73 3'UTR) were also determined in our present study, but the results of systematic experiments showed that there is no significant binding of miRNA-663b with TP73 at these two sites. The data indicated that miRNA-663b directly targets TP73 at the position 72-76 of TP73 3'UTR. To further reveal whether miRNA-663b could regulate TP73 expression in MCF-7 cells, miRNA-663b mimic and miRNA-663b inhibitor were transfected into MCF-7 cells respectively, then the transfection efficiency was detected using RT-qPCR (Fig. 2C). The protein and mRNA level of TP73 was detected by western blotting and RT-qPCR respectively. Our results indicated that miR-663b could negatively regulate TP73 expression in MCF-7 cells (Fig. 2D and E).

MiRNA-663b inhibition increases breast cancer cells TAM sensitivity. To determine whether modulation of miRNA-663b expression impacts TAM response, miRNA-663b was inhibited in the TAM resistant cell line MCF7/TAM by transfection with miRNA-663b inhibitor. Transfection efficiency was calculated by RT-qPCR (Fig. 3A). Down-regulation of miRNA-663b significantly prevented MCF7/TAM cell proliferation ability, and this inhibition was eliminated by TP73 silencing (Fig. 3B). 

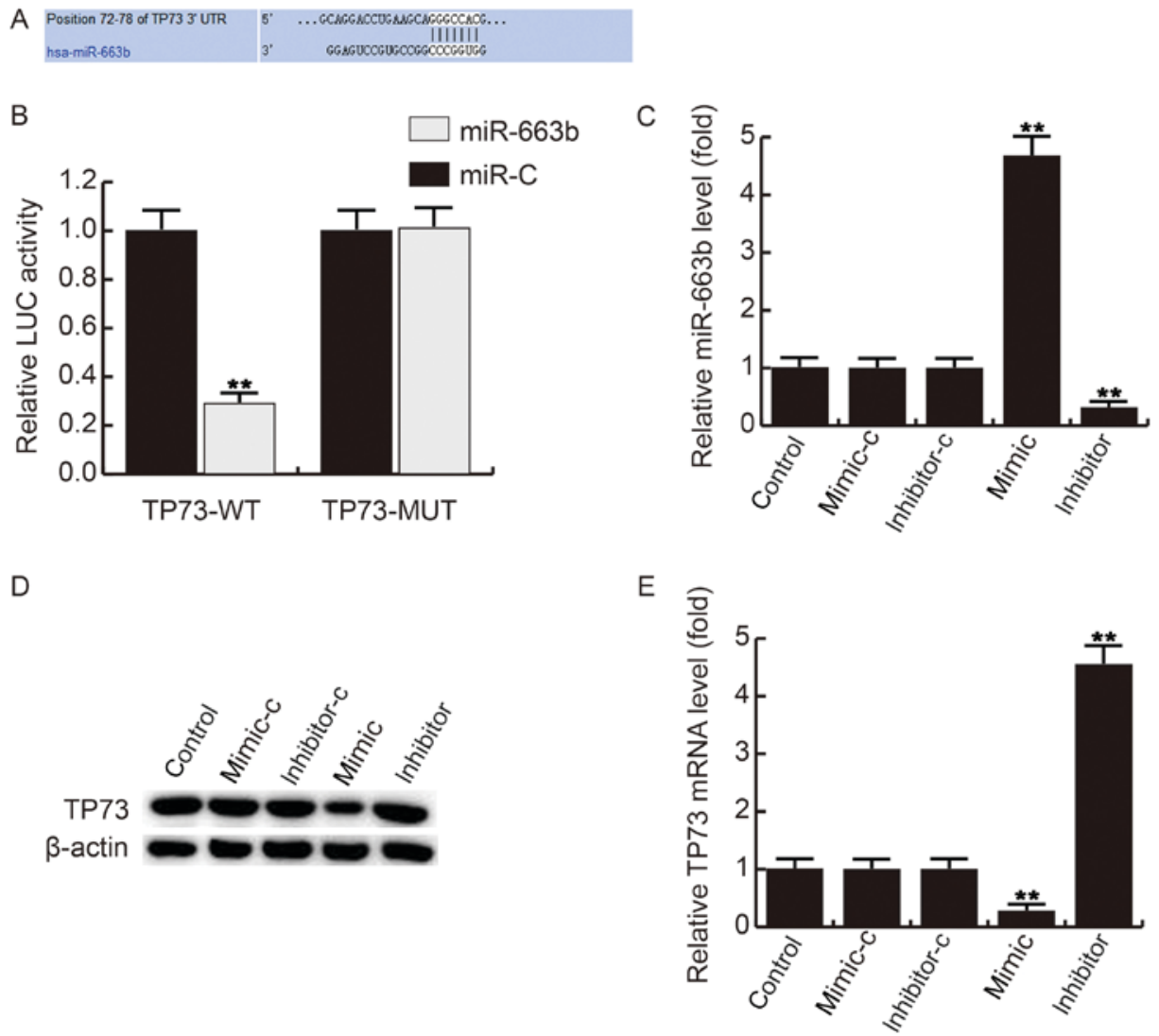

Figure 2. TP73 is a direct target of miRNA-663b. (A) Interaction between miRNA-663b and the 3'UTR of TP73 was predicted using Target Scan. (B) Luciferase activity of a reporter containing a wild-type TP73 3'UTR or a mutant TP73 3'UTR are presented. 'TP73-3'UTR-MUT' indicates the TP73 3'UTR with a mutation in the miRNA-663b binding site. ${ }^{* *} \mathrm{P}<0.01 \mathrm{vs}$. miR-C. (C) miRNA-663b expression was detected by RT-qPCR. (D) protein level of TP73 was detected using western blotting. (E) The mRNA level of TP73 was detected using RT-qPCR. Data are presented as the mean \pm standard deviation of 3 repeated experiments. ${ }^{* * *} \mathrm{P}<0.01$ vs. control. Control group, cells without any treatment; mimi-c, cells transfected with the control of miRNA-663b mimic; inhibitor-c, cells transfected with the control of miRNA-663b inhibitor; mimic, cells transfected with miRNA-663b mimic; inhibitor, cells transfected with miRNA-663b inhibitor; UTR, untranslated region; miRNA/miR, microRNA; RT-qPCR, reverse transcription-quantitative polymerase chain reaction; MUT, mutant; WT, wild type; TP73, tumor protein 73 .
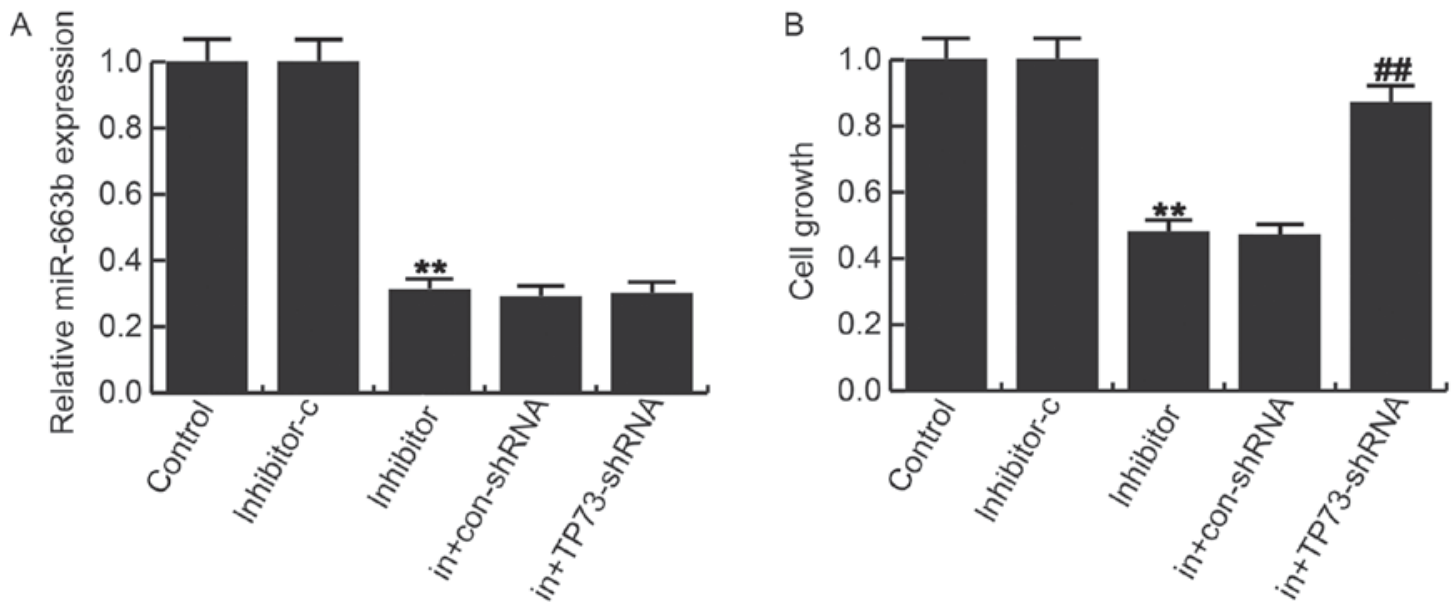

Figure 3. MiRNA-663b downregulation inhibits the proliferation in MCF7/TAM cells. (A) miRNA-663b level was detected using reverse transcription-quantitative polymerase chain reaction in the MCF7/TAM cells. (B) Cell proliferation ability was analyzed by MTT assay in the MCF7/TAM cells. Data are presented as the mean \pm standard deviation of 3 repeated experiments. ${ }^{* *} \mathrm{P}<0.01$ vs. control; ${ }^{\# \#} \mathrm{P}<0.01$ vs. inhibitor. Control group, cells without any treatment; inhibitor-c, cells transfected with the control of miRNA-663b inhibitor; inhibitor, cells transfected with miRNA-663b inhibitor; in+con-shRNA, cells co-transfected with miRNA-663b inhibitor and control-shRNA; in+TP73-shRNA, cells co-transfected with miRNA-663b inhibitor and TP73-shRNA; miRNA/miR, microRNA; shRNA, small interfering RNA; TAM, tamoxifen; TP73, tumor protein 73.

In addition, our findings showed that miRNA-663b inhibition markedly induced MCF7/TAM cell apoptosis, and TP73 silencing eliminated the effects of miRNA-663b inhibitor on MCF7/TAM cell apoptosis (Fig. 4). The data indicated 


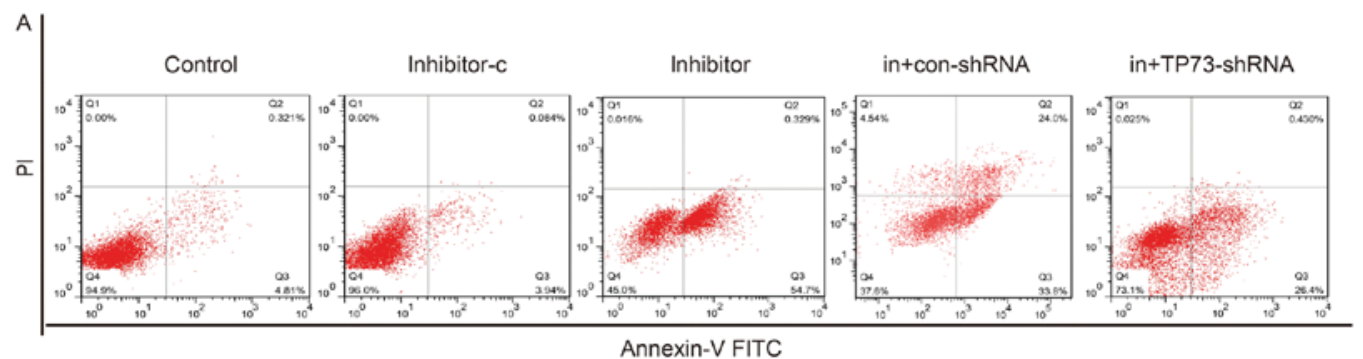

B

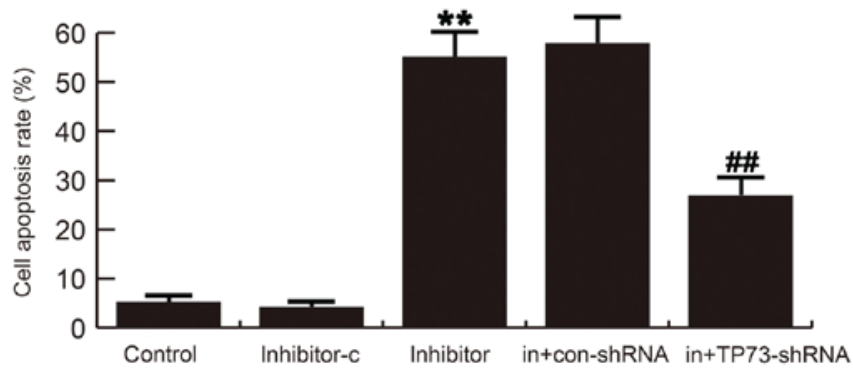

Figure 4. MiRNA-663b downregulation induces the apoptosis of MCF7/TAM cells. (A) Flow cytometry was performed to detect the cell apoptosis. (B) The data was subsequently analyzed. Data are presented as the mean \pm standard deviation of 3 repeated experiments. ${ }^{* *} \mathrm{P}<0.01$ vs. control; ${ }^{\# \#} \mathrm{P}<0.01$ vs. inhibitor. Control group, cells without any treatment; inhibitor-c, cells transfected with the control of miRNA-663b inhibitor; inhibitor, cells transfected with miRNA-663b inhibitor; in+con-shRNA, cells co-transfected with miRNA-663b inhibitor and control-shRNA; in+TP73-shRNA, cells co-transfected with miRNA-663b inhibitor and TP73-shRNA; miRNA/miR, microRNA; shRNA, small interfering RNA; TAM, tamoxifen; TP73, tumor protein 73.

A
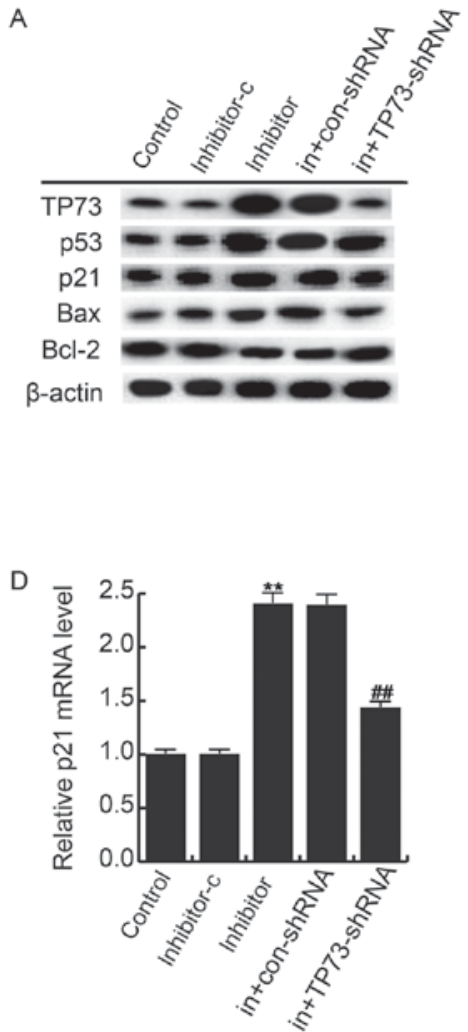

B

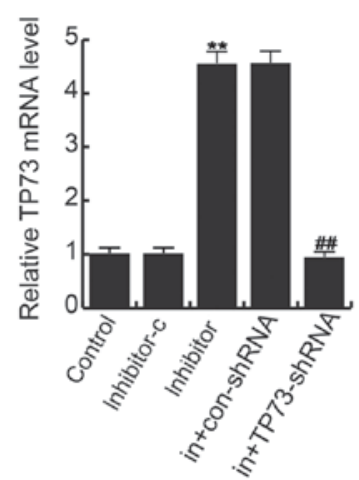

E

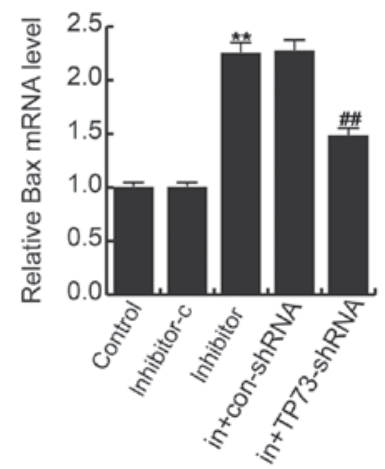

C
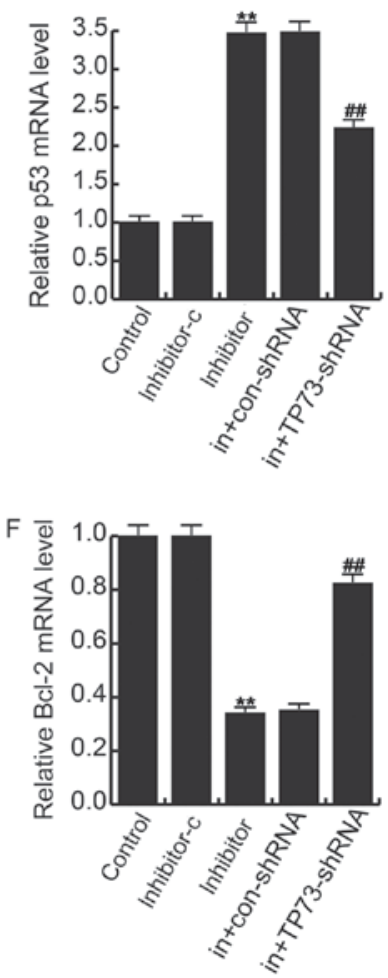

Figure 5. MiRNA-663b downregulation affects associated gene expression in MCF7/TAM cells. (A) Western blotting was performed to determine the protein expression levels of (B) TP73, (C) p53, (D) p21, (E) Bax and (F) Bcl-2 in MCF7/TAM cells. Data are presented as the mean \pm standard deviation of 3 repeated experiments. ${ }^{* *} \mathrm{P}<0.01$ vs. control; ${ }^{\# \#} \mathrm{P}<0.01$ vs. inhibitor. Control group, cells without any treatment; inhibitor-c, cells transfected with the control of miRNA-663b inhibitor; inhibitor, cells transfected with miRNA-663b inhibitor; in+con-shRNA, cells co-transfected with miRNA-663b inhibitor and control-shRNA; in+TP73-shRNA, cells co-transfected with miRNA-663b inhibitor and TP73-shRNA; miRNA/miR, microRNA; shRNA, small interfering RNA; TAM, tamoxifen; TP73, tumor protein 73; Bcl-2, B-cell lymphoma 2; Bax, Bcl-2-associated X protein.

that miRNA-663b suppression enhanced the sensitivity of MCF7/TAM cells to TAM through regulating TP73 expression.
MiRNA-663b inhibition affects the expression of $p 53, p 21, \mathrm{Bcl} 2$ and Bax in MCF7/TAM cells. Finally, the expression of cell 
growth and cell apoptosis related genes were determined in our study. As shown in Fig. 5, compared with the control group, the protein level of Bcl-2 significantly decreased in miRNA-663b inhibitor treated MCF7/TAM cells, while the protein levels of p53, p21 and Bax significantly increased. Moreover, TP73 silencing eliminated the effects of miRNA-663b inhibitor on the expression of p53, p21, Bcl2 and Bax in MCF7/TAM cells. The same trend results were obtained from the RT-qPCR analysis.

\section{Discussion}

Because of the key role of ER signaling in the development of breast cancer, blocking this pathway by endocrine therapy has become the main preventive and therapeutic approach for all stages of ER-positive breast cancer $(22,23)$. At present, there are many types of clinical endocrine therapy: i) Use of the selective estrogen receptor modulators (SERMs) that directly inhibit ER, with dual functions of competitive/antagonistic activity such as TAM; ii) use of selective ER degradation agents (SERDs) to promote ER degradation with stronger anti-estrogenic effects such as fulvestrant and iii) use of aromatase inhibitors (AI) to block the conversion of androgens to estrogens or to block estrogen production through ovarian suppression. Although endocrine therapy has a certain effect, about $30 \%$ of endocrine therapy will occur endogenous drug resistance, $40 \%$ of acquired drug resistance (18). Therefore, the emergence of endocrine therapy resistance is still a huge clinical challenge for the treatment of breast cancer.

The present study demonstrated that the expression of miRNA-663b in the TAM non-sensitive patients and TAM resistant breast cancer MCF7/TAM cells was significantly higher than that in the TAM sensitive patients and breast cancer MCF7 cells. This study reported for the first time that the down-regulation of miRNA-663b inhibited MCF7/TAM cell proliferation and induced cell apoptosis, resulting in enhanced TAM sensitivity. MiRNA-663b directly targets TP73 and negatively regulates TP73 expression. MiRNA-663b down-regulation thus indirectly enhanced p53, p21 and Bax expression, inhibited $\mathrm{Bcl}-2$ expression, which may contribute to the inhibition of MCF7/TAM cell proliferation and the induction of MCF7/TAM cell apoptosis.

At present, various miRNAs have been reported to play a key role in mediating TAM resistance in breast cancer (16-20). However, the role of miRNA-663b in TAM resistance in breast cancer remains unknown. Currently, little is known about miRNA-663b in cancer. Cai et al (24), reported that in pancreatic cancer, miRNA-663b is repressed by HOTAIR and exerts a tumor suppressor effect by targeting IGF2. MiRNA-663b may be a new circulating bio-marker for clinical diagnosis of bladder cancer (25). MiRNA-663b is over-expressed in osteosarcoma tissues (26). Human endometrial cancer patients with high miRNA-663b expression have significantly poor prognosis (27). Furthermore, miRNA-663b has been found to be a tumor suppressor in nasopharyngeal carcinoma, and it can promote nasopharyngeal carcinoma cell growth and metastasis via regulating TUSC2 (28). In the present study, we found that miRNA-663b was significantly up-regulated in TAM resistant breast cancer. We next found a novel functional link between miRNA-663b and TP73 in breast cancer MCF7 cells. TP73 (p73) is a member of the p53 family of transcription factors and was first discovered in 1997. p73 and p53 have structural and functional homology. p73 plays the role of tumor suppressor though binding and trans-activating of p53-responsive genes and inducing apoatosis and cell arrest (29). Our data also showed that miRNA-663b inhibitor significantly inhibited the proliferation and induced apoptosis of MCF7/TAM cells in vitro. $\mathrm{p} 53$ and p21 expression level markedly increased in miRNA-663b inhibited MCF7/TAM cells. Moreover, we found that the effects of miRNA-663b down-regulation on MCF7/TAM cells could be eliminated by TP73 silencing. Taken together, our results indicated that miRNA-663b inhibitor could promote the sensitivity of breast cancer cells to TAM through inducing apoptosis via targeting TP73.

In summary, we have identified miRNA-663b as an important mediator of TAM response in breast cancer.

\section{Acknowledgements}

Not applicable.

\section{Funding}

No funding was received.

\section{Availability of data and materials}

The analyzed data sets generated during the present study are available from the corresponding author on reasonable request.

\section{Authors' contributions}

HJ and LC were involved in study design, data access and analysis. PH and RL interpreted the results. All authors collaborated to develop the manuscript.

\section{Ethics approval and consent to participate}

The present study protocol was approved by the Ethics Committee of The Third Affiliated Hospital of Sun Yat-sen University, and informed consent was obtained from each patient.

\section{Consent for publication}

Not applicable.

\section{Competing interests}

The authors declare that they have no competing interests.

\section{References}

1. Vergne Y, Matta J, Morales L, Vargas W, Alvarez-Garriga C and Bayona M: Breast cancer and dna repair capacity: Association with use of multivitamin and calcium supplements. Integr Med (Encinitas) 12: 38-46, 2013.

2. Wolmark N, Mamounas EP, Baehner FL, Butler SM, Tang G, Jamshidian F, Sing AP, Shak S and Paik S: Prognostic impact of the combination of recurrence score and quantitative Estrogen Receptor Expression (ESR1) on predicting late distant recurrence risk in estrogen receptor-positive breast cancer after 5 years of tamoxifen: results from NRG Oncology/National surgical adjuvant breast and bowel project B-28 and B-14. J Clin Oncol 34: 2350-2358, 2016. 
3. Ingle JN: Postmenopausal women with hormone receptor-positive breast cancer: Balancing benefit and toxicity from aromatase inhibitors. Breast 22 (Suppl 2): S180-S183, 2013.

4. Manavalan TT, Teng Y, Appana SN, Datta S, Kalbfleisch TS, Li Y and Klinge CM: Differential expression of microRNA expression in tamoxifen-sensitive MCF-7 versus tamoxifen-resistant LY2 human breast cancer cells. Cancer Lett 313: 26-43, 2011

5. Early Breast Cancer Trialists' Collaborative Group (EBCTCG), Davies C, Godwin J, Gray R, Clarke M, Cutter D, Darby S, McGale P, Pan HC, Taylor C, et al: Relevance of breast cancer hormone receptors and other factors to the efficacy of adjuvant tamoxifen: Patient-level meta-analysis of randomised trials. Lancet 378: 771-784, 2011.

6. Early Breast Cancer Trialists' Collaborative Group (EBCTCG): Effects of chemotherapy and hormonal therapy for early breast cancer on recurrence and 15-year survival: An overview of the randomised trials. Lancet 365: 1687-1717, 2005.

7. Gutierrez MC, Detre S, Johnston S, Mohsin SK, Shou J, Allred DC, Schiff R, Osborne CK and Dowsett M: Molecular changes in tamoxifen-resistant breast cancer: Relationship between estrogen receptor, HER-2, and p38 mitogen-activated protein kinase. J Clin Oncol 23: 2469-2476, 2005.

8. Cui Y, Zhang M, Pestell R, Curran EM, Welshons WV and Fuqua SA: Phosphorylation of estrogen receptor alpha blocks its acetylation and regulates estrogen sensitivity. Cancer Res 64 9199-9208, 2004.

9. Kim VN, Han J and Siomi MC: Biogenesis of small RNAs in animals. Nat Rev Mol Cell Biol 10: 126-139, 2009.

10. Bartel DP: MicroRNAs: Target recognition and regulatory functions. Cell 136: 215-233, 2009.

11. Valencia-Sanchez MA, Liu J, Hannon GJ and Parker R: Control of translation and mRNA degradation by miRNAs and siRNAs. Genes Dev 20: 515-524, 2006.

12. Calin GA and Croce CM: Chromosomal rearrangements and microRNAs: A new cancer link with clinical implications. J Clin Invest 117: 2059-2066, 2007.

13. Iorio MV and Croce CM: MicroRNA dysregulation in cancer: Diagnostics, monitoring and therapeutics. A comprehensive review. EMBO Mol Med 9: 852, 2017.

14. Iorio MV, Ferracin M, Liu CG, Veronese A, Spizzo R, Sabbioni S, Magri E, Pedriali M, Fabbri M, Campiglio M, et al: MicroRNA gene expression deregulation in human breast cancer. Cancer Res 65: 7065-7070, 2005.

15. Volinia S, Galasso M, Sana ME, Wise TF, Palatini J, Huebner K and Croce CM: Breast cancer signatures for invasiveness and prognosis defined by deep sequencing of microRNA. Proc Natl Acad Sci USA 109: 3024-3029, 2012.

16. Yu X, Luo A, Liu Y, Wang S, Li Y, Shi W, Liu Z and Qu X MiR-214 increases the sensitivity of breast cancer cells to tamoxifen and fulvestrant through inhibition of autophagy. Mol Cancer 14: 208, 2015.
17. Shen R, Wang Y, Wang CX, Yin M, Liu HL, Chen JP, Han JQ and Wang WB: MiRNA-155 mediates TAM resistance by modulating SOCS6-STAT3 signalling pathway in breast cancer. Am J Transl Res 15: 2115-2126, 2015.

18. Cittelly DM, Das PM, Spoelstra NS, Edgerton SM, Richer JK, Thor AD and Jones FE: Downregulation of miR-342 is associated with tamoxifen resistant breast tumors. Mol Cancer 9: 317, 2010.

19. Ikeda K, Horie-Inoue K, Ueno T, Suzuki T, Sato W, Shigekawa T, Osaki A, Saeki T, Berezikov E, Mano H and Inoue S: miR-378a-3p modulates tamoxifen sensitivity in breast cancer MCF-7 cells through targeting GOLT1A. Sci Rep 5: 13170, 2015.

20. Ward A, Shukla K, Balwierz A, Soons Z, König R, Sahin O and Wiemann S: MicroRNA-519a is a novel oncomir conferring tamoxifen resistance by targeting a network of tumour-suppressor genes in ER+ breast cancer. J Pathol 233: 368-379, 2014.

21. Livak KJ and Schmittgen TD: Analysis of relative gene expression data using real-time quantitative PCR and the 2(-Delta Delta C(T)) method. Methods 25: 402-408, 2001

22. Johnston SR and Yeo B: The optimal duration of adjuvant endocrine therapy for early stage breast cancer-with what drugs and for how long? Curr Oncol Rep 16: 358, 2014.

23. Williams $\mathrm{N}$ and Harris LN: The renaissance of endocrine therapy in breast cancer. Curr Opin Obstet Gynecol 26: 41-47, 2014.

24. Cai H, An Y, Chen X, Sun D, Chen T, Peng Y, Zhu F, Jiang Y and $\mathrm{He} \mathrm{X}$ : Epigenetic inhibition of miR-663b by long non-coding RNA HOTAIR promotes pancreatic cancer cell proliferation via up-regulation of insulin-like growth factor 2 . Oncotarget 7 : 86857-86870, 2016.

25. Du M, Shi D, Yuan L, Li P, Chu H, Qin C, Yin C, Zhang Z and Wang M: Circulating miR-497 and miR-663b in plasma are potential novel biomarkers for bladder cancer. Sci Rep 5: 10437 , 2015.

26. Zhao H, Li M, Li L, Yang X, Lan G and Zhang Y: MiR-133b is down-regulated in human osteosarcoma and inhibits osteosarcoma cells proliferation, migration and invasion, and promotes apoptosis. PLoS One 8: e83571, 2013.

27. Wang YL, Shen Y, Xu JP, Han K, Zhou Y, Yang S, Yin JY, Min DL and Hu HY: Pterostilbene suppresses human endometrial cancer cells in vitro by down-regulating miR-663b. Acta Pharmacol Sin 38: 1394-1400, 2017.

28. Liang S, Zhang N, Deng Y, Chen L, Zhang Y, Zheng Z, Luo W, Lv Z, Li S and Xu T: miR-663b promotes tumor cell proliferation, migration and invasion in nasopharyngeal carcinoma through targeting TUSC2. Exp Ther Med 14: 1095-1103, 2017.

29. Casciano I, Mazzocco K, Boni L, Pagnan G, Banelli B, Allemanni G, Ponzoni M, Tonini GP and Romani M: Expression of DeltaNp73 is a molecular marker for adverse outcome in neuroblastoma patients. Cell Death Differ 9: 246-251, 2002. 